
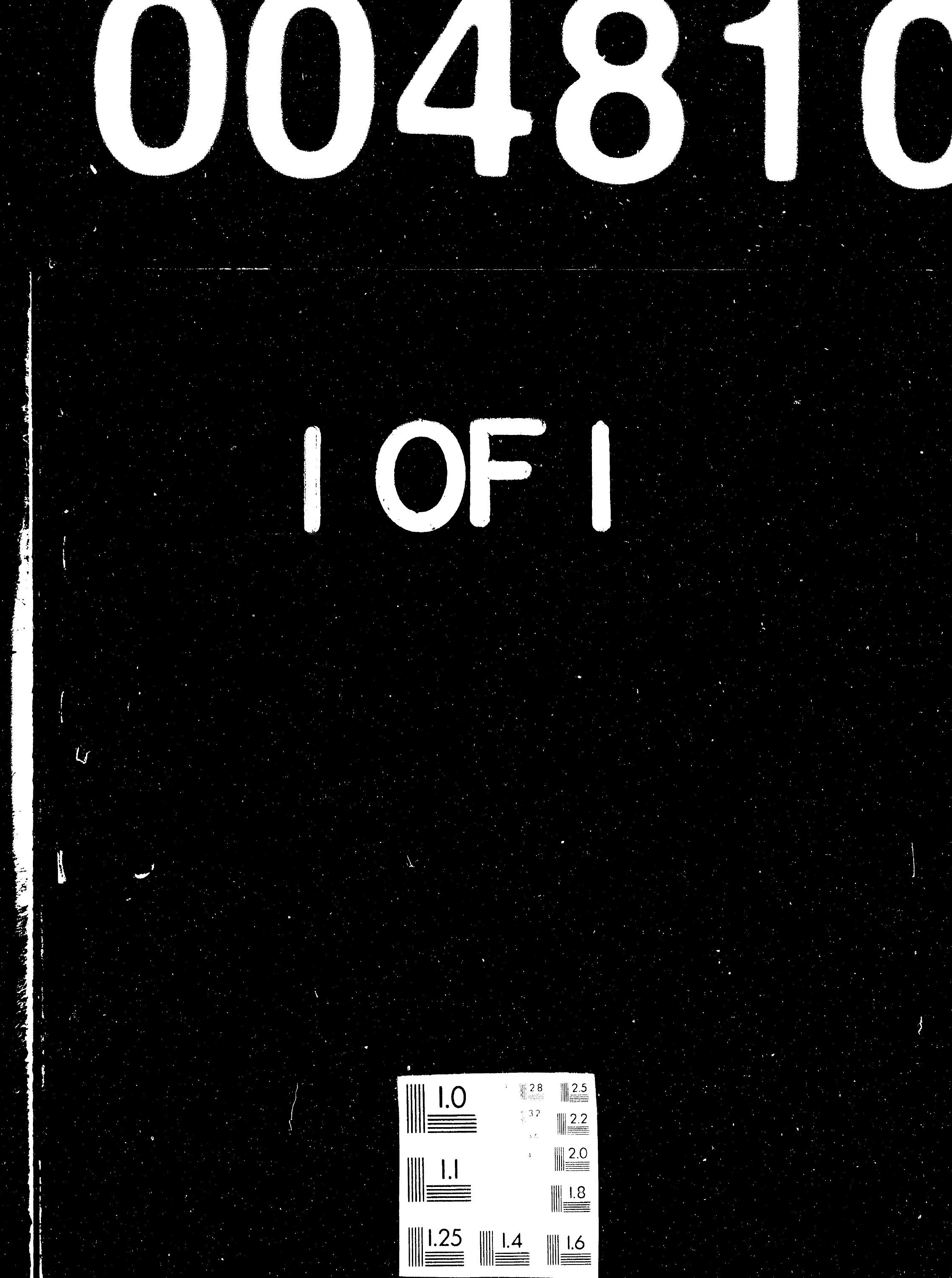
Z. Phys D (Proceedings of the Sixth International Conference on Small Particles and Inorganic Clusters, Sept. 16-22, 1992. Chicago, IL) NP, IT

ANL/CHM/CP-77993

DE93 004810

\title{
Peculiarities of structures and meltinglike transition in gold clusters*
}

\author{
I. L. Garzon ${ }^{\dagger}$ and J. Jellinek
}

Chemistry Division, Argonne National Laboratory, Argonne, Il 60439, USA

Received 16 September 1992

\section{DISCLAIMER}

\begin{abstract}
This report was prepared as an account of work sponsored by an agency of the United States Government. Neither the United States Government nor any agency thereof, nor any of their employees, makes any warranty, express or implied, or assumes any legal liability or responsibility for the accuracy, completeness, or usefulness of any information, apparatus, product, or process disclosed, or represents that its use would not infringe privately owned rights. Reference herein to any specific commercial product, process, or service by trade name, trademark. manufacturer, or otherwise does not necessarily constitute or imply its endorsement, recommendation, or favoring by the United States Government or any agency thereof. The views and opinions of authors expressed herein do not necessarily state or reflect those of the United States Government or any agency thereof.
\end{abstract}

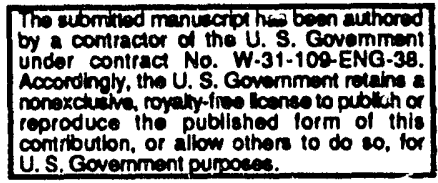

*Work performed under the auspices of the Office of Basic Energy Sciences, Division of Chemical Science, US-DOE under contract number W-31-109-ENG-38; ILG was partially supported by Project DGAPA-UNAM (IN-10-42-89) and by DGSCA-UNAM supercomputing grant

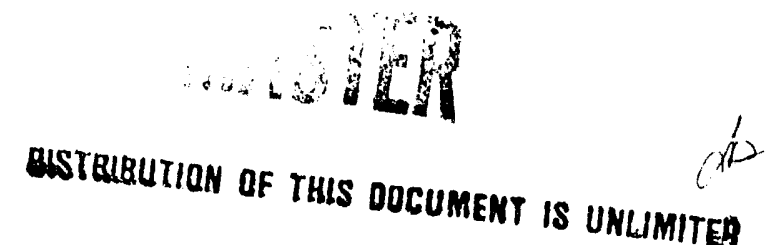




\title{
Peculiarities of structures and meltinglike transition in gold clusters*
}

\author{
I. L. Garzon ${ }^{\dagger}$ and J. Jellinek \\ Chemistry Division, Argonne National Laboratory, Argonne, il 60439, USA
}

Received 16 September 1992

\begin{abstract}
Structural and dynamical properties of gold clusters are derived from a Gupta-type potential using numerical simulations and are compared to features of nickel clusters derived from the same potential. The major implication of the comparison is that the properties of clusters mimicked by this many-body potential depend, in general, on the values of its parameters.
\end{abstract}

PACS: 36.40

\section{Introduction}

One of the important issues of theoretical studies of atomic and molecular clusters is the question of how the different types of bonding (van der Waals, ionic, covalent and metallic) exhibit themselves through specific structural and dynamical features of these systems [1]. The bulk of the studies is based on numerical simulations in which the interatomic interactions are represented by parameterized potential energy functions. Both the functional form of the potential and the values of the parameters play, in general, an important role. In the case of the Lennard-Jones potential, for example, the dependence on the element (material) can be eliminated by using reduced units of energy and distance. As a consequence, the qualitative features of the structure(s) and dynamics of clusters of different sizes deduced from this potential are generic and valid for an entire class of elements: an example is clusters of noble gases. Potentials for non-van der Waals systems usually depend on more than two parameters, and they do not satisfy the

\footnotetext{
*Work performed under the auspices of the Office of Basic Energy Sciences, Division of Chemical Science, US-DOE under contract number W-31-109-ENG-38; ILG was partially supported by Project DGAPA-UNAM (IN-10-42-89) and by DGSCA-UNAM supercomputing grant

† Permanent address: Instituo de Fisica, Universidad Nacional Autónoma de México, Apartado Postal 2681, 22800 Ensenada, Baja California, Mexico
}

principle of the corresponding states. Changing the values of the parameters in these potentials alters the topology of the corresponding potential energy surfaces and thereby affects both the geometries (the number of the different isomers and their energy ordering) and the dynamics of the clusters. Analyses for additive pairwise interactions have been given recently [2].

Here we present results on the structures and dynamics of the meltinglike transition in gold clusters derived from a Gupta-type potential; cf. Refs. 3-5. These are compared with predictions obtained from the same potential but using parameters appropriate for nickel [6] (cf. also Ref. 7). The potential and the methodology are sketched in section 2 . The results and their discussion are given in section 3.

\section{Potential and methodology}

We use the Gupta-type potential $[4,6]$ which in reduced units of energy $V^{*}$ and interatomic distances $r_{i j}^{*}$ is written as

$$
V^{*}=\frac{1}{2} \sum_{j=1}^{n}\left\{A \sum_{i=1}^{n} \exp \left(-p\left(r_{i j}^{*}-1\right)\right)-\left[\sum_{i=1}^{n} \exp \left(-2 q\left(r_{i j}^{*}-1\right)\right)\right]^{1 / 2}\right\}
$$

In Eq. ( 1 ) $n$ is the number of acoms considered, $p=10.15$ and $q=4.13$ are chosen as appropriate for gold [8], and the value $A=0.118438$ is determined to yield the minimum energy fcc structure of the bulk gold [4]. The reduced potential energy $V^{*}$ and interatomic distances $r_{i j}^{*}$ are related to their corresponding absolute magnitudes $V$ and $r_{i j}$ through $V^{*}=V / U_{n}$ and $r_{i j}^{*}=r_{i j} / r_{\text {on }}$, where the "units" of energy $U_{n}$ and of distance $r_{\text {on }}$ depend on the element (material) and on the size (n) of the cluster [6].

The dynamics of the clusters at different fixed total energies are generated by solving numerically Newton's equations of motion for all the atoms. The Verlet propagator [9] with a step size of $1.43 \cdot 10^{-15}$ s is used. The initial conditions are chosen in such a way that the clusters do not translate and/or rotate. Even in the longest runs ( $5 \cdot 10^{6}$ 


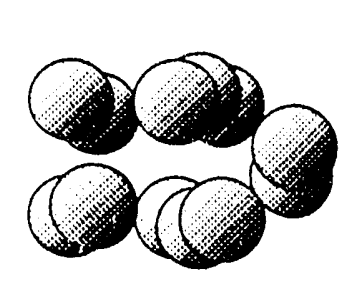

$-10.804$

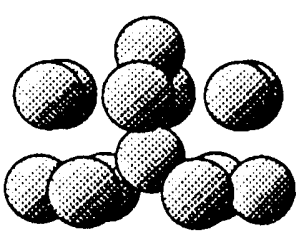

$-12.745$

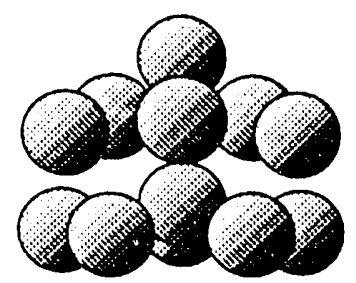

$-10.790$

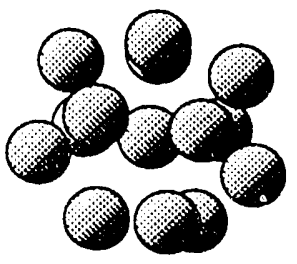

.12 .711

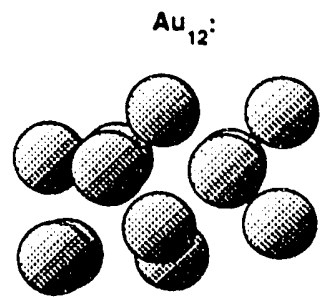

$-10.788$

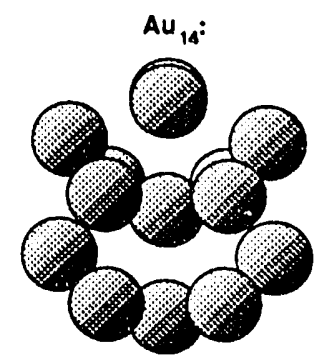

$-12.705$

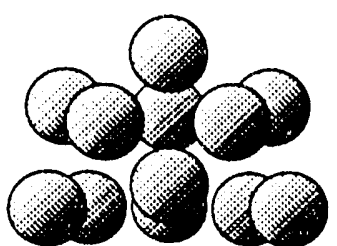

$-10.769$

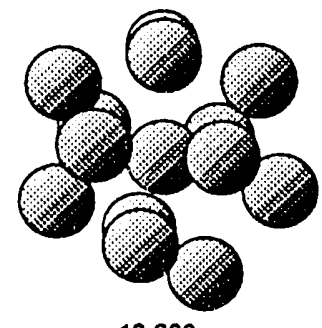

.12 .699

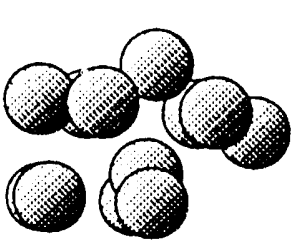

$-10.761$

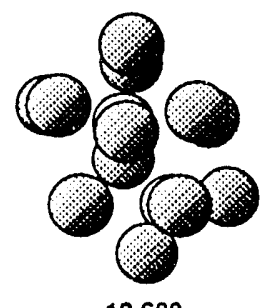

$-12.688$

Fig. 1. Isomers and their energies (in reduced units) of the $A u_{12}$ and $A u_{14}$ clusters.

steps) the total energy is conserved within $1.4 \%$. The structures of the clusters corresponding to minima of the potential energy surface are obtained by quenching the (internal) kinetic energy of the clusters.

\section{Results and discussion}

Detailed calculations were carried out for clusters of $n=12$, 14,19 and 20 atoms. These complement the results for $n=$ 6,7 and 13 atoms published earlier [4]. We illustrate here our new findings through the comparison of the structural and dynamical properties of $\mathrm{Au}_{12}$ and $\mathrm{Au}_{14}$ with those of $\mathrm{Ni}_{12}$ and $\mathrm{Ni}_{14}$; a complete account and analysis of the results will be given elsewhere.

Figure 1 displays low energy structures of the $\mathrm{Au}_{12}$ and $\mathrm{Au}_{14}$ clusters. For both the low-lying isomers are close in energy (in relative units), albeit the gap between the first two isomers of $\mathrm{Au}_{14}$ is larger than that of $\mathrm{Au}_{12}$ even on the per atom basis. The structures of $\mathrm{Au}_{14}$ are, with a possible exception of the most stable isomer, more spherical than those of $\mathrm{Au}_{12}$. One notices that the lowest energy isomers of $\mathrm{Au}_{12}$ and of $\mathrm{Au}_{14}$ (as obtained in our thermal quenching simulations) are very different from the characteristic fivefold icosahedral packing notwithstanding the fact that the icosahedron is the most stable geometry of $\mathrm{Au}_{13}$ [4]. In contrast, the most stable structures of $\mathrm{Ni}_{12}$ and of $\mathrm{Ni}_{14}$ (Fig. 2), generated from the same potential (1) but with the parameters $A=0.101036, p=9$ and $q=3$ appropriate for nickel, are the (relaxed) structures obtained from a 13-atom icosahedron (the lowest energy isomer of $\mathrm{Ni}_{13}$ ) by removing a surface atom and adding an extra atom over a three-fold face, respectively [6]. The structural differences between the most stable isomers of gold and nickel clusters are well illustrated by the distributions of the bond lengths shown in Fig. 3. The transparent tendency toward a more uniform distribution of the bond length in gold clusters is characteristic not only for $n=12$ and 14 , but also for clusters of larger sizes.
The evolution of the dynamics of the gold clusters with an increase of their (internal) energy (per atom) reflects a typical meltinglike transition $[1,4,6,7]$. This transition involves the so-called "coexistence" stage, i.e., a size-dependent finite range of energies (per atom) for which the cluster undergoes spontaneous isomerization transitions in its time evolution. The different isomers survive long enough on the time scale defined by characteristic vibrational period of the cluster to be identified as such. Figure 4 displays the root-mean-square (rms) bond length fluctuation $\delta$,

$\delta=\frac{2}{n(n-1)} \sum_{i<j}^{n} \frac{\left(\left\langle r_{i j}^{2}\right\rangle-\left\langle r_{i j}\right\rangle^{2}\right)^{1 / 2}}{<r_{i j}>}$.

as a function of the cluster temperature $T$,

$\mathrm{T}=\frac{2\left\langle E_{k}\right\rangle}{(3 n-6) k}$.

for $\mathrm{Au}_{12}$ and $\mathrm{Au}_{14}$. (In the above equations, $\mathrm{r}_{\mathrm{ij}}$ is the distance between atoms $i$ and $j, E_{k}$ is the kinetic energy of the cluster, $k$ is the Boltzmann constant, and $<>$ denotes time-averaging.) The abrupt increase in the corresponding

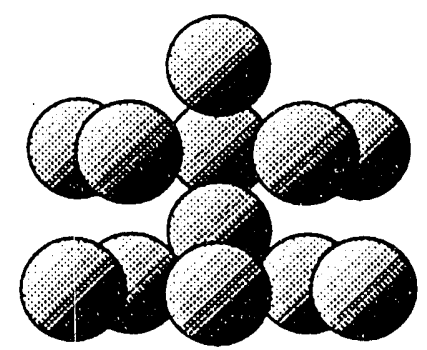

$\mathrm{Ni}_{12}: \mathrm{E}=-11.292$

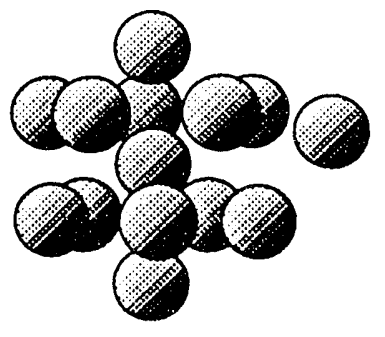

$\mathrm{Ni}_{14}: \mathrm{E}=-13.398$
Fig. 2. The most stable isomers and their energies (in reduced units) of the $\mathrm{Ni}_{12}$ and $\mathrm{Ni}_{14}$ clusters. 


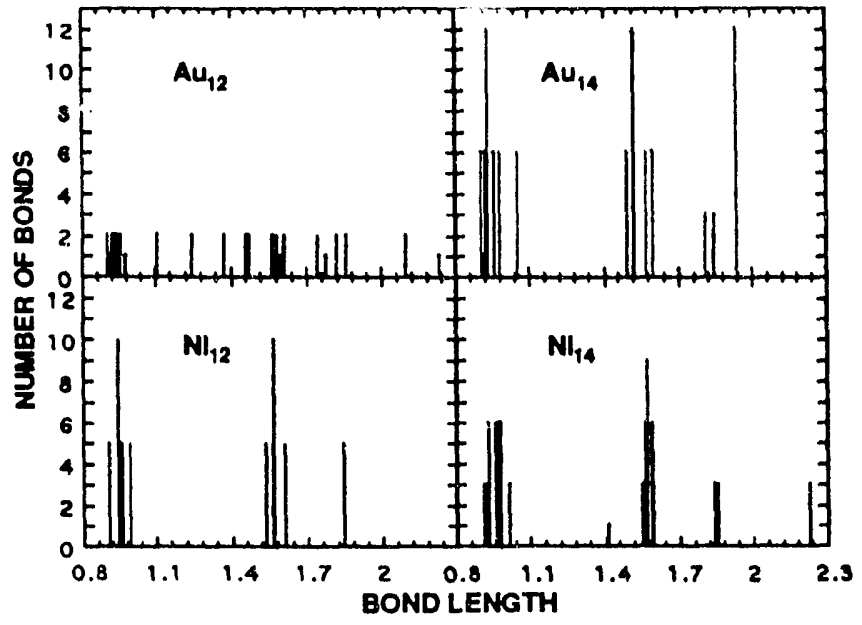

Fig. 3. Distribution of bond lengths in the most stable isomers of the $\mathrm{Au}_{12}, \mathrm{Au}_{14}, \mathrm{Ni}_{12}$ and $\mathrm{Ni}_{14}$ clusters.

values of $\delta$ is the signature of the "melting" in these clusters. Using the procedure described in Ref. 4 we obtain $\mathrm{U}_{12}=3.156 \mathrm{eV}$ for $\mathrm{Au}_{12}$ and $\mathrm{U}_{14}=3.215 \mathrm{eV}$ for $\mathrm{Au}_{14}$. Defining the "melting temperature" of the clusters as the value of $T$ corresponding to $\delta=0.18$ (approximately the middle of the transition range) and using the estimated values of the size-dependent units of energy $U_{12}$ and $U_{14}$ we arrive at the absolute "melting temperatures" of about $460 \mathrm{~K}$ for $\mathrm{Au}_{12}$ and $440 \mathrm{~K}$ for $\mathrm{Au}_{14}$. These can be compared with the "melting temperature" of $-680 \mathrm{~K}$ for $\mathrm{Au}_{13}$ [4].

The comparison with the melting properties of $\mathrm{Ni}_{12}$ and $\mathrm{Ni}_{14}$ leads to two important observations. First, similar to the case of $\mathrm{Ni}_{12}$ (see Fig. 3 in Ref. 6a) the meltinglike transition in $\mathrm{Au}_{12}$ exhibits itself as a single abrupt rise in the corresponding $\delta$ graph. By fitting the energy of the most stable structure of $\mathrm{Ni}_{12}$ to the value obtained from an embedded-atom potential [7], we obtain $\mathrm{U}_{12}=3.231 \mathrm{eV}$, which puts the absolute melting temperature of this cluster (defined by the same criterion $\delta=0.18$ ) at $-1200 \mathrm{~K}$ (cf. Ref. 7). The relative reduction in the "melting temperatures" of the 12-atom gold and nickel clusters from the corresponding bulk values ( $1338 \mathrm{~K}$ for gold and $1726 \mathrm{~K}$ for nickel) is quite different: it is larger for $\mathrm{Au}_{12}$ by a factor of -2 . An even larger difference has been obtained for $\mathrm{Au}_{13}$ and $\mathrm{Ni}_{13}$ [4]. Second, the $\delta$ plot for $\mathrm{Au}_{14}$ exhibits also only a single abrupt rise as a function of the cluster temperature, while that for $\mathrm{Ni}_{14}$ is characterized by two such rises corresponding to the "premelting" (local melting) and the (complete) "melting" transitions [6a,7]. The solid-to-liquidlike transition in $\mathrm{Au}_{14}$ does not involve the "premelting" stage. A partial reason for this is that the lowest energy structures of the $\mathrm{Au}_{14}$ and $\mathrm{Ni}_{14}$ clusters are very different.

In summary, we presented results on the properties of gold clusters derived from a Gupta-type potential and compared those with our earlier findings for nickel clusters derived from the same potential. The main implication of the comparison is that both the structural and the dynamical properties of clusters modelled by this many-body potential depend, in general, on its parameters. This conclusion complements similar findings obtained for pairwise-additive interactions [2]. A more complete account and analysis of the results will be the subject of future publications.

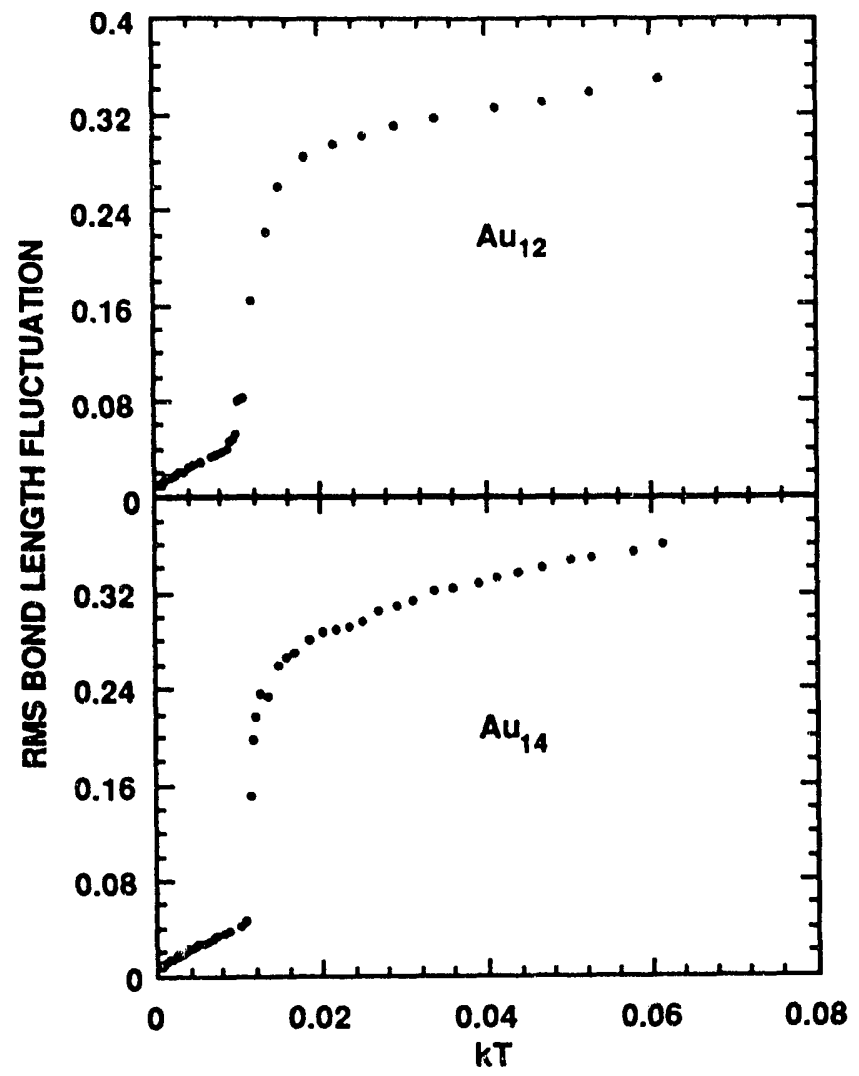

Fig. 4. Rms bond length fluctuations as a function of $\mathrm{kT}$ (in reduced units) for $\mathrm{Au}_{12}$ and $\mathrm{Au}_{14}$.

\section{References}

1. See, for example, in: Adv. Chem. Phys. Prigogine, I., Rice, S.A. (eds.). Vol. 10, Part 2. New York, Wiley-Interscience 1988; Physics and Chemisiry of Finite Systems: From Clusters to Crystals. Jena, P., Khanna, S.N., Rao, B.K. (eds.). Vols. 1-2. Dordrecht: Kluwer Academic Publishers 1992;; and referencas therein

2. Braier, P.A., Berry, R.S., Wales, D.J.: J. Chem. Phys. 93. 8745 (1990); Stillinger, F.H., Stillinger, D.K.: ibid 93. 6106 (1990); Chang, H.P., Berry, R.S.: ibid 97, 3573 (1992)

3. Gupta, R.P.: Phys. Rev. B23, 6265 (1981); Sawada, S., Sugano, S.: Z. Phys. D - Atoms, Molecules and Clusters 14, 247 (1989)

4. Garzon, I.L., Jellinek, J.: Z. Phys. D - Atoms, Molecules and Clusters 20, 235 (1991)

5. Ercolessi, F., Andreoni, W., Tossatti, E.: Phys. Rev. Lett. 66, 911 (1991); Lin, M.E., Ramachandra, A.. Andres, R.P., Reifenberger, R.: Physics and Chemistry of Finite Systems: From Clusters to Crystals. Jena, P., Khanna, S.N.. Rao, B.K. (eds.), Vol. 1, p. 309. Dordrecht: Kluwer Academic Publishers 1992

6. a) Jellinek, J., Garzon, I.L.: Z. Phys. D - Atoms, Molecules and Clusters 20, 239 (1991); b) Garzon, I.L., Jellinek. J.: Physics and Chemistry of Finite Systems: From Clusters to Crystals. Jena, P., Khanna, S.N., Rao, B.K. (eds.), Vol. 1, p. 405. Dordrech:: Kluwer Academic Publishers 1992

7. Guvenç, Z.B., Jellinek, J.. Voter, A.F.: Physics and Chemistry of Finite Systems: From Clusters to Crystals. Jena, P., Khanna, S.N., Rao, B.K. (eds.), Vol. 1, p. 411. Dordrecht: Kluwer Academic Publishers 1992

8. Rosato, V.. Guillope, M., Legrand, B.: Philos. Mag. A59. 321 (1989)

9. Verlet, L.: Phys. Rev. 159. 98 (1967) 

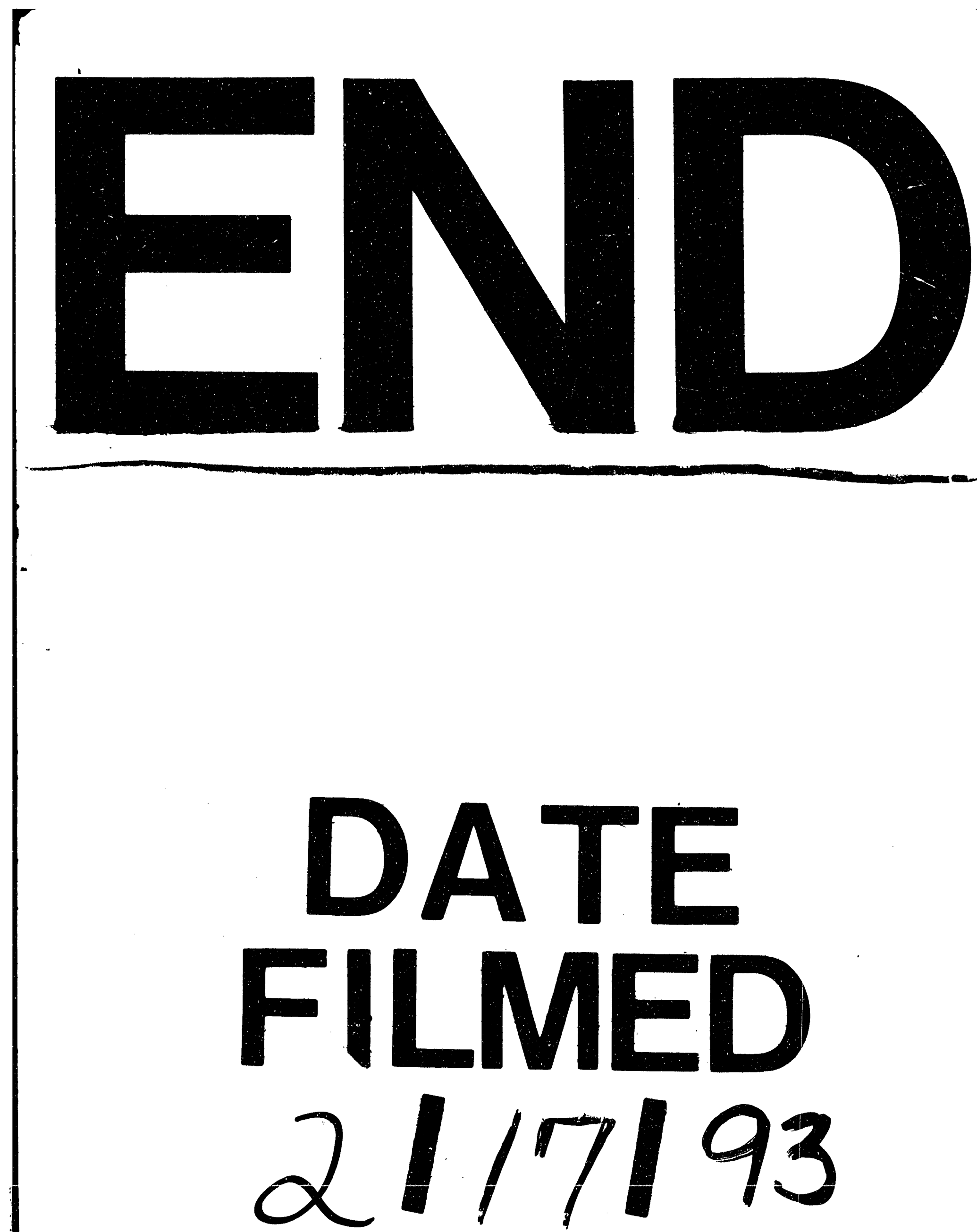
